\title{
Increase in Role of Destructive Analysis in a New Nuclear Era
}

\author{
Yusuke Kuno \\ Nuclear Non-Proliferation Science and Technology Centre, \\ Japan Atomic Energy Agency (JAEA) \\ (Nuclear Engineering and Management, University of Tokyo)
}

\begin{abstract}
Keywords: Non-proliferation, Nuclear Materials, Accountancy, Safeguards, Destructive Analysis, DA,
\end{abstract} Environmental Sampling

\section{ABSTRACT}

Since many clandestine nuclear activities in the Middle East, the Korean peninsula and other areas of the world have been disclosed during the last 15 years, a series of the counter-measures have been proposed and taken. The Destructive Analysis (DA) for environmental sampling for safeguards (ESS), based on the Additional Protocol to the NPT Safeguards Agreement has played very important role since 1990s. It is designed to detect nuclear materials in the environment of a facility that may reveal the presence of an undeclared nuclear activity such as plutonium recovery from irradiated fuel or isotopic enrichment of uranium.

Among the verification tools for the comprehensive Safeguards agreement, DA for nuclear material accountancy and its verification is extremely important for drawing quantitative Safeguards conclusions. In particular, DA is the best approach for detecting "bias defects", which arise when small amounts of nuclear material are diverted over a protracted length of time. Nuclear accountancy and verification based on the DA with a state-of-the-art determination technique providing highest possible measurement accuracy is the fundamental and essential technology, without which Safeguards cannot be concluded. Timely and more accurate analytical services are current challenges against getting larger scale of nuclear fuel cycle.

This paper describes the role of DA for both environmental sampling and verification of nuclear material accountancy.

\section{INTRODUCTION}

World-wide concerns about the proliferation of nuclear materials/technologies have considerably increased with the realization of some states' or possibly terrorists' clandestine attempts at production of nuclear weapons, whereas there are greater demands for peaceful use of nuclear materials in the present time. The problem on nuclear proliferation has been approached with the combination of the following measures;

- Strengthening Safeguards (increase in capabilities of IAEA inspection under the Protocol additional to
Comprehensive Safeguards Agreement) under the existing NPT regime.

- $\quad$ Strengthening non-proliferation of nuclear weapons through the implementation of Guidelines for nuclear exports and nuclear related exports by Nuclear Suppliers Group (NSG)

- Strengthening Physical Protections; adoption of the revised Convention on the Physical Protection of Nuclear Material in 2005.

- Counter-proliferation of weapons of mass destruction by Proliferation Security Initiative (PSI)

- Efforts towards conclusion of Comprehensive Nuclear-Test-Ban Treaty (CTBT) and Fissile Material Cut-off Treaty (FMCT).

- IAEA's proposed Multilateral Nuclear Approaches (MNA) on nuclear material supplies, and

- President Bush's Plan on international supplies of nuclear materials to reduce proliferation risks.

A fundamental system to seek permanent solutions should be based upon internationally agreed measures, which can be widely and effectively implemented. In this context, the currently established Safeguards system of the IAEA, consisting of comprehensive safeguards and the additional protocol should represent the strongest system presently in force for deterrence of nuclear proliferation. Although it may not be directly effective to the non-NPT states, there is no other immediate and established measure which can effectively stop increasing the number of countries who intend to clandestinely develop nuclear weapon.

Among the tools that the IAEA presently uses for Safeguards assurance, nuclear Destructive Analysis (DA) is extremely important for drawing Safeguards conclusions. "Environmental sampling for Safeguards (ESS)" was added to the Safeguards arsenal in 1996. ESS is a powerful tool for gaining confidence that illicit nuclear weapon capabilities are not developed clandestinely in contravention of a State's non-proliferation commitments. ESS is designed to detect traces of nuclear materials in the environment of a facility that may reveal the presence of an undeclared nuclear activity such as plutonium recovery from irradiated fuel or isotopic enrichment of uranium. DA of environmental samples has been internationally 
recognised as a strong tool for drawing safeguards conclusions based on the experience gained during the UN-sanctioned inspections in Iraq in the 1990's.

Improvement of quality and technical level of DA can be a very effective manner for States to promote peaceful use of nuclear materials because clear declaration based on accurate accountancy of nuclear material is a fundamental and unavoidable manner to gain reliance from the international community. IAEA inspectors make independent measurements to verify the amount of nuclear materials presented by States. This is in line with Article 55 of INFCIRC/153, stating "the system of measurements on which the records used for the preparation of reports are based shall either conform to the latest international standards or be equivalent in quality to such standards".

The role of the existing DA currently used for the Safeguards is reviewed, whereas a study for future contributions in DA is also made in this study.

\section{DESTRUCTIVE ANALYSIS FOR SAFEGUARDS IN IAEA AND JAPAN}

General idea on the role of DA can briefly

described as Fig. 1.

Regarding verification for nuclear material accountancy, DA combines state-of-the-art determination techniques having the highest possible accuracy, e.g. in accordance with ITV2000 [1]. This is particularly necessary for detecting "bias defects" which arise when small amounts of nuclear material are diverted over a protracted length of time.

DA involving chemical and physical analyses is the only method to accomplish this important role. DA is performed for

the purposes of verifying the quality and the effectiveness of the Operator's measurement system, as well as for estimating random and systematic errors which are necessary to judge the significance of material unaccounted for (MUF).

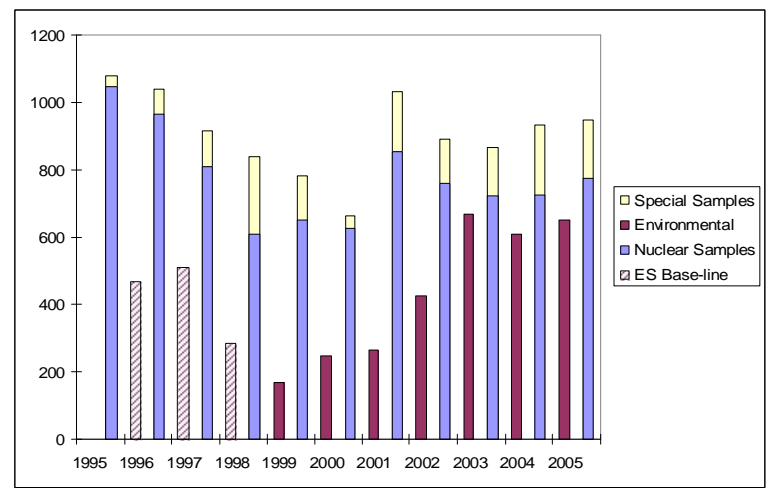

Fig. 2 Trend on IAEA Safeguards Samples
Namely, equivalently high accuracy in destructive analytical level is also demanded for operator's side. In other words operator's accountancy system has to be performed by the highest level of quality. In this context, the Japanese analytical laboratories for reprocessing and plutonium fuel fabrication in JAEA have attempted to improve their quality for the last 2 decades, while the Safeguards analytical laboratories of the IAEA and NMCC (Nuclear Material Control Centre, the State's Analytical party, Japan) have played the important role for verification.

The analytical capabilities of IAEA have been extended into the area for Environmental Sampling for Safeguards (ESS) since the start of its Clean Laboratory's operation in 1996. There is need to extend analytical capacity and capabilities for ESS because of remarkable increase in environmental analytical services in IAEA (Fig. 2). A NWAL (network of analytical laboratories) for ESS has been established not only for increase of capacity to provide additional throughput to cope with peak sample loads but also additional capabilities to apply analytical methods that the SAL does not posses, and parallel

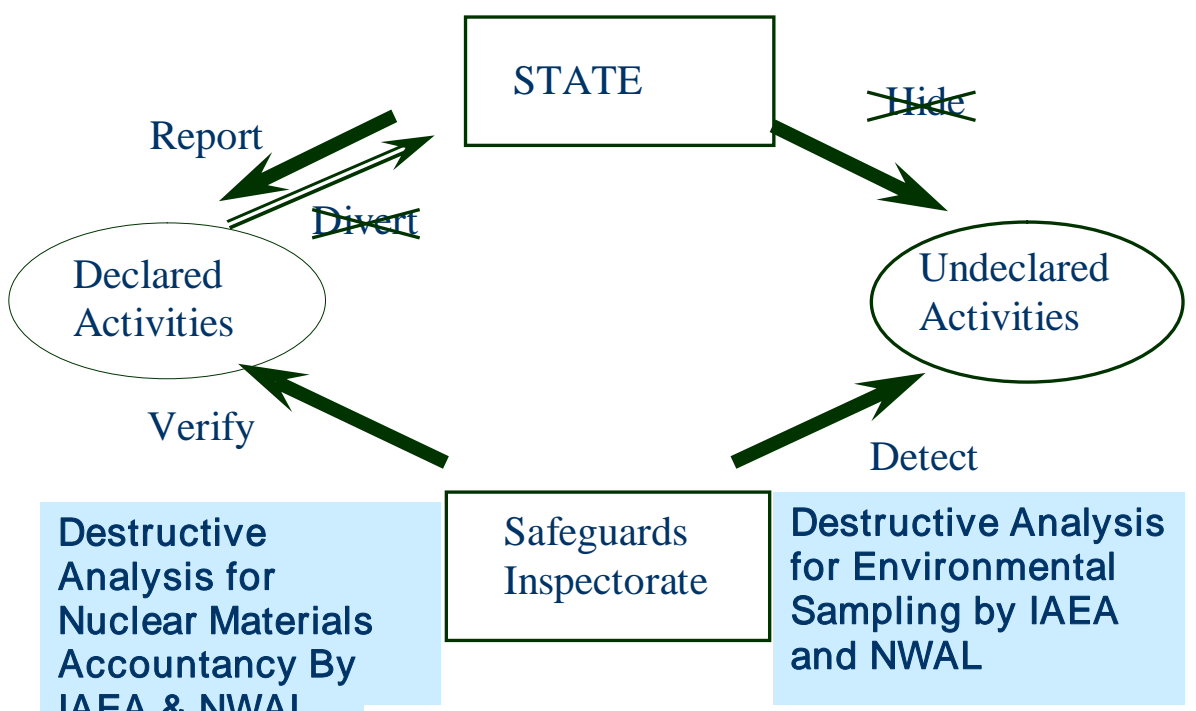

Fig. 1 General Role of DA in Safeguards Inspection

analysis of samples to increase confidence in the accuracy of the results, but also to pursue reliable analytical results by plural laboratories (Fig. 3) [2,3].

Fig.3 IAEA-NWAL for Environmental Sampling

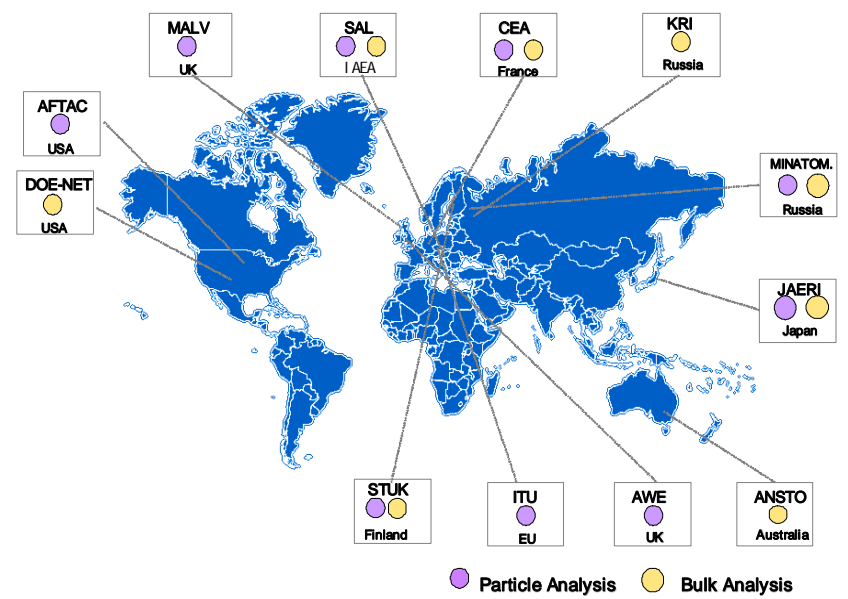

Copyright @ 2007 by JSME 


\author{
MALV : Particle-SIMS \\ ITU : Particle-SIMS \\ CEA : Particle-SIMS, Bulk-TIMS Particle-(FT)-TIMS, \\ J AEA : Particle-SIMS, Bulk-ICPMS \\ MINATOM : Particle-SIMS, Bulk-TIMS \\ AFTAC : Particle-(FT)-TIMS \\ STUK : Particle-SIMS, Bulk-TIMS \\ AWE : Particle-(FT)-TIMS \\ ANSTO : Accelerator-MS \\ SANES : (ORNL, LANL, LLNL, PNNL, SRTC) Bulk-analysis \\ KRI : Bulk-TIMS \\ IAEA-SAL : Screening, SEM/XRF, Particle-SIMS, Bulk-TIMS
}

A clean-room analytical facility with cleanliness of ISO class 5 has been established in JAEA in Japan, which has met IAEA' qualification on ESS and joined IAEA-NWAL in 2003 [4].

\section{CURRENT STATUS OF SAFEGUARDS DESTRUCTIVE ANALYSIS}

\subsection{DA for Nuclear Materials}

Table 1 shows uncertainties for typical nuclear material

Table 1 Basic Techniques and Their Uncertainties in Nuclear Material Sample Analysis at IAEA-SAL ( ): International Target Values [ITV2000]

measurements (weight/volume) and Containment and Severance (CS). Protracted diversion, however, may not possibly be detected by the above-mentioned ones. Time necessary for detection of such protracted one should be dependent on throughput of nuclear production facility of interest. Namely, larger in the scale of nuclear fuel cycle, more timely DA is required for both inspector's and operator's sides. On-Site-Laboratory (OSL) at a large scale reprocessing plant is a new challenge for the conventional DA field [6] because it is not sufficient for Safeguards timelines criterion to perform analysis of its verification samples at the central IAEA laboratory-SAL in Austria (spending 40-60 days for DA-input/Pu, 40-50 days for shipment of samples) [7].

The IAEA and the Japanese Safeguards authority agreed in the early 1990s on the establishment of a Joint On-Site Laboratory (OSL) within the Japan Nuclear Fuel Limited (JNFL) facility located at Rokkasho-Mura, Japan. Features of the OSL is as follows;

- Verification sensitivity: on site measurements do not require the extensive sample treatments necessary for plutonium shipments from Japan, nor the sample size limitations required under the Japanese transport regulation,

- On site measurements facilitate arrangements to

\begin{tabular}{lll}
\hline & Random, Relative \% & $\begin{array}{l}\text { Systematic, } \\
\text { Relative } \%\end{array}$ \\
\hline $\begin{array}{l}\text { U-235 by Mass-Spectrometry } \\
\text { e.g. 1-2\% enriched }\end{array}$ & $0.05(0.1)$ & $0.05(0.1)$ \\
\hline U-235 by Gamma Spectrometry & 0.15 & 0.15 \\
\hline U by Titration & $0.08(0.1)$ & $0.1(0.1)$ \\
\hline U by Gravimetry & $0.05(0.05)$ & $0.05(0.05)$ \\
\hline $\begin{array}{l}\text { U by Isotopic Dilution } \\
\text { Spectrometry (IDMS) }\end{array}$ & $0.1(0.2)$ & $0.1(0.2)$ \\
\hline Pu by Titration & & $0.1(0.1)$ \\
\hline Pu by Coulometry & $0.08(0.1)$ & $0.1(0.1)$ \\
\hline Pu by IDMS & $0.05(0.1)$ & $0.1(0.2)$ \\
\hline
\end{tabular}

verification analyses that the IAEA-SAL (Safeguards Analytical Laboratory) has employed [5]. It is obvious that all the analytical methods shown here for verification of operator's accountancy, have achieved the high level of quality required by International Target Values (ITV) 2000 [1]. For the verification of "Bias Defect Analysis", namely to verify operator's measurement system in an efficient and effective manner, it is essential that Operator's performance level, quality of operator's measurement system, is as good as inspector's one. Tokai Reprocessing Plant of JAEA, for example, has performed destructive analysis of input accountancy solutions with $0.07 \%$ systematic error and $0.01 \%$ random error from 1994 through 2002, whereas IAEA also performed it with very small uncertainties such a level of less than $0.1 \%$ for both systematic and random errors for the same period.

Gross and partial defect can be detected by a combination of some measures available on sites, such as Non-Destructive Assay (NDA), density measurement, bulk ensure authenticity of sample, thereby supporting

verification integrity, Timeliness: having on-site

measurements mean that results will be available within a few days. That capability will mean that analytical measurements can be used promptly in deriving conclusions for timeliness purposes,

- Safety: performing the measurements at the OSL will reduce the prospects for sample losses or accidents.

Establishment of the authentication measures is essential for the On-Site Laboratory (OSL) at the Rokkasho Reprocessing Plant (RRP) where IAEA's and the state's Safeguards analytical authorities have to share analytical operation. An image of the OSL of RRP is given in Photo 1, whereas the thorough authentication measures are introduced [8]. 


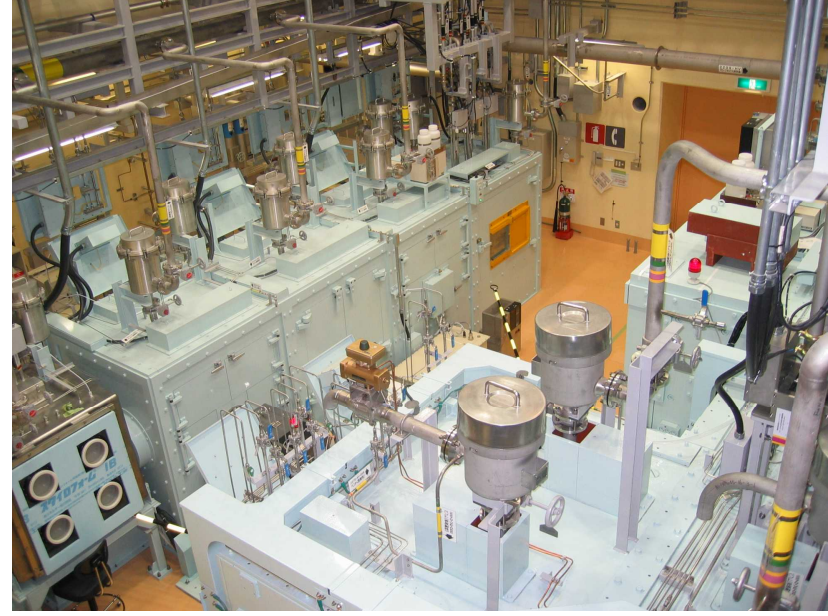

Photo 1. The OSL of RRP

\subsection{DA for Environmental Sampling}

IAEA inspectors collect environmental samples at facilities all over the world and measurements are performed by the IAEA-SAL and the NWAL. The SAL provides certified ultra-pure sampling materials, mainly cotton swipe/hot-cell swipe (Photo 2) to inspectors, receives them after inspections, screens and splits and redistributes subsamples to the NWAL without significant risk of crosscontamination. Quality control (QC) samples are also distributed to the NWAL in parallel [2].

Collected swipe samples undergo screening at the IAEASAL Clean Laboratory, flowed by detailed analysis, bulk analysis and/or particle analysis. Table 2 shows a summary of the major methods for analysis of environmental samples employed at the SAL and NWAL.

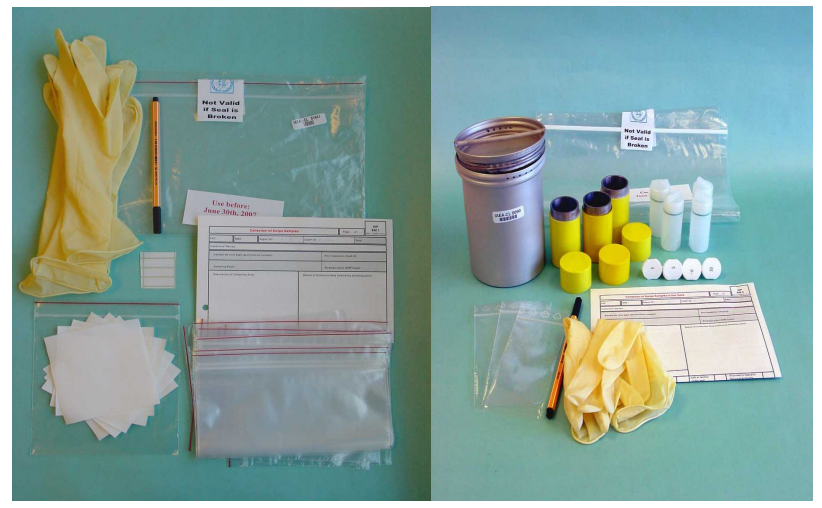

Photo 2 Representative Sampling Kits Employed by IAEA

SIMS is one of the standard analytical tools for particle analysis to find and measure particles of interest. The feature of the SIMS is its ability to quickly search millions of particles to find the ones of interest, followed by individual isotopic measurements with a micro beam, using an electron multiplier detector. It achieves the highest transmission and detector sensitivity. The total efficiency (number of ions collected in the detector(s) vs. number of atoms in the particle) for micro

beam measurement for 0.4 to $1 \mu \mathrm{m}$ size particles consisting of approximately $400 \mathrm{fg}$ to $6 \mathrm{pg}$ of uranium, which makes the efficiency in the measurements critical, is for uranium oxide about 0.2-0.3\% [9]. The scanning electron microscope (SEM) equipped with both energydispersive (ED) and wavelength-dispersive (WD) X-ray fluorescence spectrometers (XRF) plays an important role for the elemental analysis of particles taken from environmental swipe samples to estimate chemical composition of each particle [10]. Features of analytical methods for Environmental Sampling are also summarised in Table 2.

Table 2 Current Analytical Methods for Environmental Sampling

\begin{tabular}{|c|c|c|}
\hline & Purposes & Characteristics of method \\
\hline $\begin{array}{l}\text { XRF, HRGS } \\
\text { Screening }\end{array}$ & Screening & $\begin{array}{l}\text { To confirm location and } \\
\text { approx. amount of of } U \text { and } \\
\mathrm{Pu} \text {, presence of } \mathrm{FP}\end{array}$ \\
\hline $\begin{array}{l}\text { Chemical } \\
\text { separation- } \\
\text { TIMS/ } \\
\text { ICPMS }\end{array}$ & $\begin{array}{l}\text { Bulk } \\
\text { isotopic }\end{array}$ & $\begin{array}{l}\text { To obtain average } \\
\text { information of nuclear } \\
\text { materials (isotopic, amount) } \\
\text { with very high sensitivity; } \\
\text { U:10-100 pg, Pu several fg }\end{array}$ \\
\hline$\overline{\mathrm{ANM}}$ & $\begin{array}{l}\text { Bulk } \\
\text { isotopic }\end{array}$ & $\begin{array}{l}\text { To obtain minor isotopics } \\
\text { accurately U-236/U-238 } \\
\text { ratio }<10^{-8}, 10 \text { fg Puで } 10 \% \\
\text { RSD }\end{array}$ \\
\hline SIMS & $\begin{array}{l}\text { Particle } \\
\text { isotopic }\end{array}$ & $\begin{array}{l}\text { To obtain specific isotopic } \\
\text { information of individual } \\
\text { particle in a rapid manner } \\
\text { (1-2 days): Less accuracy for } \\
\text { minor isotopics }\end{array}$ \\
\hline SEM/XRF & $\begin{array}{l}\text { Particle } \\
\text { elemental }\end{array}$ & $\begin{array}{l}\text { To obtain elemental } \\
\text { information of individual } \\
\text { particle (estimate chemical } \\
\text { compositions) }\end{array}$ \\
\hline $\begin{array}{l}\text { SEM/XRF - } \\
\text { ICPMS or } \\
\text { TIMS }\end{array}$ & $\begin{array}{l}\text { Particle } \\
\text { isotopic }\end{array}$ & $\begin{array}{l}\text { To obtain specific isotopic } \\
\text { information of individual } \\
\text { particle in accurate manner } \\
\text { also for minor isotopics }\end{array}$ \\
\hline $\begin{array}{l}\text { Fission } \\
\text { Track -TIMS }\end{array}$ & $\begin{array}{l}\text { Particle } \\
\text { isotopic }\end{array}$ & $\begin{array}{l}\text { To obtain specific isotopic } \\
\text { information of individual } \\
\text { particle in a rapid manner } \\
\text { (1-2 days) in accurate and } \\
\text { reliable for minor isotopics. } \\
\text { Time-consuming. 1\% RSD } \\
\text { on U-235/U-238, 5\% RSD } \\
\text { on U-236/U-238 }\end{array}$ \\
\hline
\end{tabular}




\section{FUTURE DESTRUCTIVE ANALYSIS FOR SAFEGUARDS}

\subsection{DA for Nuclear Materials}

The overall objective of Safeguards is to provide credible assurance of both the non-diversion of nuclear material from declared activities and the absence of undeclared nuclear material and activities in a State as a whole. In order to draw a SG conclusion that all nuclear material in the State with a comprehensive safeguards agreement and an additional protocol in force has been placed under Safeguards and remained in peaceful nuclear activities or has been otherwise adequately accounted for, the Agency must draw conclusions of both the non diversion of declared nuclear material and the absence of undeclared nuclear material and activities.

For such a conclusion, nuclear material accountancy remains fundamental measure. The IAEA's current practice of evaluating the nuclear material balance annually for all types of nuclear material is therefore also retained in spite of the international requirement of more effective and efficient nuclear verification [12]

For accountancy in the future fuel nuclear cycle such as FBR cycle, where more $\mathrm{Pu}$ throughput may be treated, operator's analysis has to provide more accurate results for its declaration than the present situation because larger MUF is expected. The $\sigma_{\text {MUF }}$ (entire yearly error of accountancy system) can be improved by a combination of several measures. One should be the reduction of the systematic errors in both the measurement systems of operator's and inspector's, which is heavily dependent of the employment of more sophisticated design for nuclear accountancy system such as ideal accountancy tank + representative sampling system from complete homogenous solutions, small inner-plant inventory etc. Reduction of DA systematic error may still not be impossible. Assuming the realisation of more accurate DA in operator's side, particularly with less systematic error, smaller On-SiteLaboratory (OSL) than RRP's can be sufficient if the systematic errors are improved. Nevertheless, larger in the scale of nuclear fuel cycle, more timely DA is required for both inspector's and operator's sides, as stated earlier. The role of OSL is getting to be more important than centralised analytical activities at IAEA-SAL.

Systematic errors may be equipment-intrinsic once tank/device/equipment are designed and installed. Namely it is dependent on calibration method. Randomization on calibration of equipment, e.g. to randomize short-term systematic errors by calibration with different independent reference material for each analytical method, more frequent calibration with monitoring systematic error with different analytical technique would be an idea for the improvement. More careful calibration on balk measurement may also be required for future accountancy. Table 3 shows a list of errors for key measurement points of a reprocessing accountancy, given by ITVs [1]. A typical large scale reprocessing plant that has input and output batches of $20 \mathrm{~kg}$ and $80 \mathrm{~kg}$ (Pu-sizes), respectively, is taken as a study for accountancy error. The bottom line of the table gives total uncertainties calculated. The flow $\sigma_{\mathrm{MUF}}$ (yearly) can be estimated $15.3 \mathrm{~kg}$ in this example. If systematic error of each error factor can be reduced by $0.1 \%$, although it may not be easy, $\sigma_{\mathrm{MUF}}$ may be calculated as $7.7 \mathrm{~kg}$. This implies that efforts to improve uncertainties for conventional accountancy system may still be able to provide significant and dominant contribution for better accountancy and safeguards system of future large scale nuclear fuel cycles.

Table 3 Measurement Errors for Reprocessing Accountancy

\begin{tabular}{|l|c|c|c|c|}
\hline $\begin{array}{l}\text { Error } \\
\text { factor }\end{array}$ & \multicolumn{2}{|l|}{$\begin{array}{l}\text { Input Pu (dissolver } \\
\text { solution) }\end{array}$} & \multicolumn{2}{l|}{$\begin{array}{l}\text { Output Pu (U/Pu- } \\
\text { mixed) }\end{array}$} \\
\hline & $\begin{array}{l}\text { Random, } \\
\text { Relative } \\
\%\end{array}$ & $\begin{array}{l}\text { Systematic } \\
\text { Relative \% }\end{array}$ & $\begin{array}{l}\text { Random, } \\
\text { Relative } \\
\%\end{array}$ & $\begin{array}{l}\text { Systematic } \\
\text { Relative \% }\end{array}$ \\
\hline Vol. & 0.3 & 0.2 & 0.20 & 0.2 \\
\hline Sampling & 0.3 & 0.2 & 0.2 & $\mathrm{Nd}^{1)}$ \\
\hline Pu-conc. & $\begin{array}{c}0.2 \\
\text { (IDMS) }\end{array}$ & $\begin{array}{c}0.2 \\
\text { (IDMS) }\end{array}$ & $\begin{array}{c}0.15 \\
\text { (IDMS) }\end{array}$ & $\begin{array}{c}0.15 \\
(\text { IDMS) }\end{array}$ \\
\hline Total (\%) & \multicolumn{2}{|c|}{$0.35^{2)}$} & \multicolumn{2}{c|}{$0.32^{2)}$} \\
& \multicolumn{2}{|c|}{} \\
\hline
\end{tabular}

1) $0.2 \%$ is assumed.

2) 160 and 40 batches are assumed, respectively, for the calculation.

Samples from the recent Complementary Access (CA) include many nuclear samples (271 samples in 2004 [11]) requiring analysis of $\mathrm{U}, \mathrm{Pu}, \mathrm{Th}, \mathrm{Am}$, other elements/nuclides and isotopic determinations (major/minor isotopes) in a variety of matrices, determinations of the chemical form in organic and inorganic compounds. The analysis of $\mathrm{Cm}$ in highly radioactive samples from nuclear reprocessing has recently started for the verification of plutonium in hulls and high level radioactive waste in bitumen. Analytical capabilities for measuring $\mathrm{Np}$ and Am should be ready in response to the needs for verification of Alternate Nuclear Materials (ANM) [13]. Analytical capabilities for measuring $\mathrm{Np}$ and Am should be ready in response to the needs for verification of Alternate Nuclear Materials (ANM) [13].

\section{2 DA for Environmental Sampling}

So-called 'special' analytical needs shown in Fig 2, associated mainly with Complementary Access (CA), have turned out to be one of the major challenges in recent years for DA for Safeguards verification. The total number of special samples that IAEA acquires in both the nuclear and environmental categories has significantly increased in 2004-5[11]. The nature of analytical samples for CA appears to be a mixture of environmental and nuclear grade materials, containing complex matrices. Although some relevant methods such as elemental analysis in various different matrices (Inductively Coupled Plasma mass Spectrometry;ICPMS), identification of organic compounds 
(Fourier Transform Infrared Spectroscopy; FTIR, GasChromatography; GC, Direct Chemical Ionization Mass Spectrometry; DCI-MS), determination of cation/anion in solution samples (Ion-chromatograph) and morphology / chemical form of sample materials, are still either under study. Applying DA for investigating the origin or age of materials is a new challenge. This could be the area to which Japan can contributes in future, particularly for the samples from Asian countries in terms of the effective use of its highly advanced analytical capabilities and the reduction of time necessary for the transportation.

One of the most critical point required for environmental analysis may be 'rapid' analytical services. Average (median) time required for DA analytical services (shipment + analysis + evaluation) of high priority samples is 3 months, whereas, time for routine priority samples is 11-13 months according to 2003/4 IAEA annual report. The IAEA's Network of the Analytical Laboratories (NWAL) for the environmental analysis has been extended for recent years. Although the capacity and capability of the ES NWAL is currently meeting the relevant requirements of IAEA in general, the capacity for sensitive particle analyses, particularly in terms of fulfilment of timeliness requirement, should desirably be increased[14]. Contribution from Japan is highly expected by IAEA in this area.

Extensive analytical capabilities are required in the measurement of minor isotopics for uranium, i.e. U-236 and $\mathrm{U}-234$ in a rapid manner to identify the source of the uranium acquired. It should be as rapid as SIMS and as accurate as Fission Track (FT) TIMS. Implementation of Ultra-High Sensitive SIMS (UHS-SIMS) would be an option for this requirement, which may be capable of providing results comparable to FT-TIMS in uranium particle analysis (including minor isotopes) in much faster manner than FT-TIMS. Satisfactory performance was confirmed by an experiment with UHS-SIMS in U-234/U235 and U-236/U-235 [15-16]. Fig. 4-1 gives present distribution of IAEA's environmental sample analyses, where approx. 1/3 of analysis samples are measured by FTTIMS for mainly accurate $\mathrm{U}$ isotopic analysis, $1 / 3$ by SIMS for rapid confirmation of U-235, and $1 / 3$ for bulk analysis. Those may not have completely fulfilled Safeguards requirement, i.e. rapid and accurate analysis including minor isotopics. If UHS-SIMS is introduced to the environmental Safeguards analysis, dramatic change in NWAL's regime may be expected. Fig. 4-2 gives such an expected future composition of analytical method for environmental analysis.

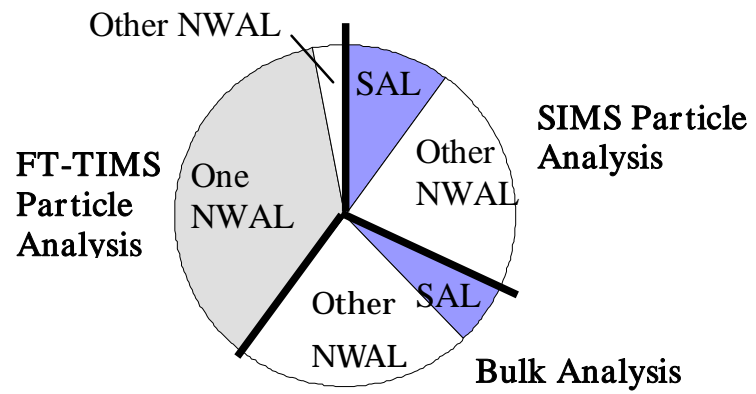

Fig. 4-1 Present Compositions of Environmental Analysis

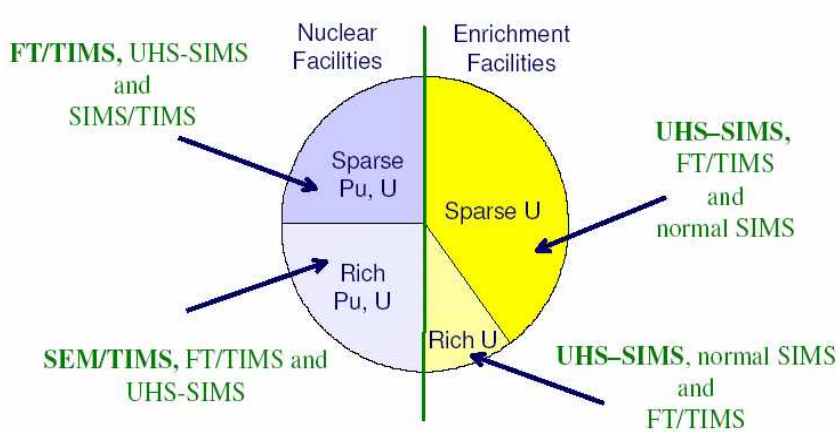

Fig. 4-2 An optimised future use of analytical methods (Suggested primary methods in bold, followed by complementary method)

There are considerable differences in the volume (throughput) of analytical services among the NWAL laboratories, in particular too much dependent on one specific laboratory in FT-TIMS, in spite of the fact that the number of NWAL participants have increased in recent years. More even and wider distribution of analytical samples in the NWAL system should be realized by introducing UHS-SIMS, as also observed in Fig. 4-2.

Based on the above discussion, the following is expected to Japan for analysis of the environmental sampling;

1) Japan, as a country supporting International Safeguards strongly, should cover a considerable portion (e.g. 1/4) of IAEA's analytical needs, since Japan possesses one of the largest clean laboratories of IAEA NWAL, which has already been equipped with stateof-the-art analytical techniques and potentially capable of providing high amount of analytical services at very high quality,

2) The proposed method, UHS-SIMS, should be introduced to the Safeguards' field of Japan to reinforce the entire IAEA capabilities because very few NWAL have so far been prepared for this option in spite of its promising technology.

3) Japan should continue to be a pioneer country for the development of new environmental analysis techniques as it has led development some remarkable ones such as vacuum particle preparation method, low uranium content swipe material. A new challenge of the picking-up-particles has been progressing in Japan in advance of the other NWALs.

4) Japanese FT-TIMS should immediately participate in IAEA NWAL particle analysis since it is getting close to the technical completion.

The remaining issues in analysis for environmental sampling would be rapid and reliable determination of $\mathrm{Pu}$ in particles and confirmation of the presence of $\mathrm{Pu}$ and $\mathrm{Am}$ at 
lower concentrations than the current detection limit and isotopic and determination of elements other than $\mathrm{U}$ and $\mathrm{Pu}$ in a micrometer-sized particle can be a challenge of obtaining more information from a limited amount of nuclear material in a sample.

\section{CONCLUSION}

DA has played a very important role for Safeguards verification under comprehensive SG agreements and the Additional Protocol. The overall requirements for IAEADA have remarkably changed in recent years. Improvement in IAEA DA regime and capabilities as described in this paper should be considered in order to cope more effectively and efficiently with the current requirements of the world community.

For nuclear material analysis;

$>\quad$ The most sophisticated measures in nuclear material accountancy should be considered from the initial stage of design for the future and the next generation nuclear fuel cycle. In particular, improvement in measurement uncertainties, even in DA techniques, should first be examined.

$>$ Local verification laboratory activities such as OSL may become more important than centralised IAEA laboratory for the nuclear material verification because of the new era of larger scale fuel cycles. In this context, Japan might have to play an important role.

For analysis of environmental sampling;

$>$ More timely and accurate analytical services are required, e.g., for uranium minor isotopics.

$>$ More even and wider distribution of analytical samples in the NWAL system should be realized.

$>$ Japan, as a technically-advanced country in DA for the environmental sampling programme, should make more significant contribution in this area, e.g. to cover a considerable portion of IAEA's analytical needs since it has already had one of the largest and the most reliable NWAL.

$>$ Japan should continue to be a pioneer for the development of new environmental analysis techniques including some promising techniques such as UHS-SIMS.

$>$ Japanese FT-TIMS should take part in IAEA NWAL particle analysis to reinforce the entire IAEA capabilities and capacity.

\section{REFERENCES}

[1] H.Aigner et al.: "International Target Values 2000 for Measurement Uncertainties in Safeguards Nuclear Materials", STR-327, IAEA, April 2001.

[2] Y.Kuno, et al.: "An Update on SAL Analytical Capabilities for Safeguards Environmental Sampling", Proc. of INMM 43 ${ }^{\text {rd }}$ Annual Meeting, Phoenix, July 2003.

[3] Y.Kuno: Nuclear Eye, Vol. 51, No. 4 p 25, 2005.

[4] Y.Hanzawa et al. : J. Science and Technology, Vol. 40, No.1 p 49 (2004)

[5] Y.Kuno: "The role of Destructive Analysis for Surveillance of Nuclear Non-proliferation", Vol. 1, 2003, p 11, Bunseki.
[6] G.Duhamel et al.: "Establishing the Joint IAEA/JSGO/NMCC Safeguards On Site Laboratory for the Rokkasho Reprocessing Plant": Major Challenge for managing the interfaces" Proc. Int. Conf on Facility Operations - Safeguards Interface, Charleston, USA, February/March 2004.

[7] P.Doherty et al.: NWAL Support to IAEA Safeguards Analysis", Symposium on International Safeguards,

Vienna, October 2001.

[8] G.Duhamel et al. "Establishing the Joint

IAEA/JSGO/NMCC Safeguards On Site Laboratory for the Rokkasho Reprocessing Plant”, GLOBAL 2005, October 2005, Tsukuba, Japan.

[9] K.Ingeneri et al.: "Enhanced Uranium Ionization Efficiency for Thermal Ionization Mass Spectrometry Using Cavity Ion Source", Proc. of INMM 42 ${ }^{\text {nd }}$ Annual Meeting, Indian Wells, July 2001.

[10] A.Ciurapinski, et al.; "Particle Analysis for a Strengthened Safeguards System, Use of SEM equipped with EDXRF and WDXRF", J. Radioanal. and Nucl. Chem. Vol 251 No.3, 345.

[11] Y.Kuno et al.: "Increase in the Role of Destructive Analysis for Safeguards Verification", GLOBAL 2005, October 2005, Tsukuba, Japan.

[12] "Safeguards System of the IAEA", Safeguards, IAEA Homepage: www.iaea.org/

[13]Y.Kuno, et al.: "Development of Analytical Techniques for U/Pu/Np/Am at the Safeguards Analytical Laboratory", Proc. IAEA Safeguards Symposium, Vienna, Austria, October/November 2001

[14] Discussion at IAEA SAL Workshop, 27-30 Nov 2006.

[15] M.Hedberg et al.: "Isotopic Measurements of U particles by Secondary Ionisation Mass Spectrometry (SIMS), Proc. of INMM 46 ${ }^{\text {th }}$ Annual Meeting, July 2005.

[16] Private communication with M.Hedberg. 